\title{
Bayesian mixture modeling for spectral density estimation
}

\author{
Annalisa Cadonna ${ }^{\mathrm{a}, *}$, Athanasios Kottas ${ }^{\mathrm{a}}$, Raquel Prado ${ }^{\mathrm{a}}$ \\ ${ }^{a}$ Department of Applied Mathematics and Statistics, University of California at Santa Cruz \\ 1156 High Street, Santa Cruz, California 95064, U.S.A.
}

\begin{abstract}
We develop a Bayesian modeling approach for spectral densities built from a local Gaussian mixture approximation to the Whittle log-likelihood. The implied model for the log-spectral density is a mixture of linear functions with frequency-dependent logistic weights, which allows for general shapes for smooth spectral densities. The proposed approach facilitates efficient posterior simulation as it casts the spectral density estimation problem in a mixture modeling framework for density estimation. The methodology is illustrated with synthetic and real data sets.

Keywords: Logistic mixture weights; Markov chain Monte Carlo; Normal mixtures;

Whittle likelihood.
\end{abstract}

\section{Introduction}

Spectral density estimation from multiple observed time series is important in fields where information about frequency behavior is relevant, such as neuroscience, econometrics and geoscience. For example, electrical signals measuring brain activity, such as electroencephalograms, are typically recorded at multiple locations over the scalp of a given subject in neuroscience studies. In addition, such studies often involve several subjects and various treatments or experimental conditions. Therefore, providing flexible methods for spectral analysis of multiple time series is key, particularly since the methods that are currently available in the literature cannot be easily extended to deal with these cases.

* Corresponding author

Email addresses: acadonna@soe.ucsc.edu (Annalisa Cadonna), thanos@soe. ucsc.edu (Athanasios Kottas), raquel@soe.ucsc.edu (Raquel Prado)

Preprint submitted to Journal of ${ } T_{E} X$ Templates

December 2, 2016

C) 2017. This manuscript version is made available under the Elsevier user license http://www.elsevier.com/open-access/userlicense/1.0/ 
We develop a mixture modeling approach for a single spectral density, which is amenable to extensions for hierarchical modeling of multiple spectral densities. Throughout, we assume $n$ realizations $x_{1}, \ldots, x_{n}$ from a zero-mean stationary time series $\left\{X_{t}: t=1,2, \ldots\right\}$, with absolutely summable autocovariance function $\gamma(\cdot)$. The spectral density function is defined as

$$
f(\omega)=\sum_{k=-\infty}^{+\infty} \gamma(k) \exp (-i k \omega), \quad \text { for }-\pi \leq \omega \leq \pi,
$$

where $\gamma(k)=\mathrm{E}\left(X_{t+k} X_{t}\right)$ denotes the autocovariance function. An estimator of the spectral density is the periodogram, defined as $I_{n}(\omega)=\left|\sum_{t=1}^{n} x_{t} \exp (-i t \omega)\right|^{2} / n$. Although $I_{n}(\omega)$ is defined for all $\omega \in[-\pi, \pi]$, it is computed at the Fourier frequencies $\omega_{j}=2 \pi j / n$, for $j=0, \ldots,\lfloor n / 2\rfloor$, where $\lfloor n / 2\rfloor$ is the largest integer not greater than $n / 2$. Because of the symmetry of the periodogram, there are only $\lfloor n / 2\rfloor+1$ effective observations. In fact, following common practice, we exclude the observations at $\omega_{j}=0, \pi$, and therefore the sample size is $N=\lfloor n / 2\rfloor-1$. It is well known that the periodogram is not a consistent estimator of the spectral density. Consistent estimators have been obtained by smoothing the periodogram or the log-periodogram through windowing methods, as in, for instance, [1].

Model-based approaches to spectral density estimation are typically built from the Whittle likelihood approximation to the periodogram [2]. For relatively large sample sizes, the periodogram realizations at the Fourier frequencies, $I_{n}\left(\omega_{j}\right)$, can be considered independent. In addition, for large $n$ and for zero-mean Gaussian time series, the $I_{n}\left(\omega_{j}\right)$, for $\omega_{j} \neq 0, \pi$, can be considered independent exponentially distributed with mean $f(\omega)$. The main advantage of the Whittle likelihood with respect to the true likelihood is that the spectral density appears explicitly and not through the autocovariance function. Moreover, under the Whittle likelihood, the estimation problem can be cast in a regression framework, with observations given by the log-periodogram ordinates and regression function defined by the log-spectral density: $\log \left(I_{n}\left(\omega_{j}\right)\right)=\log \left(f\left(\omega_{j}\right)\right)+\epsilon_{j}$, for $j=1, \ldots, N$. Here, the $\epsilon_{j}$ follow a log-exponential distribution with scale parameter 1 . In this context, frequentist estimation approaches include approximating the distribution of the $\epsilon_{j}$ with a normal distribution and fitting a smoothing spline to the log-periodogram [3], and maximizing the Whittle likelihood with a roughness penalty 
term [4]. Bayesian approaches have also been developed. [5] approximate the distribution of the $\epsilon_{j}$ with a mixture of normal distributions and assign a smoothing prior to $\log (f(\omega))$. [6] express the log-spectral density as $\log (f(\omega))=\alpha_{0}+\alpha_{1} \omega+h(\omega)$, with a Gaussian process prior on $h(\omega)$. A different approach based on Bernstein polynomial priors was considered by [7], with emphasis on posterior consistency results. [8] propose Bayesian wavelet-based smoothing of the log-periodogram.

We present a new Bayesian approach to spectral density estimation. Building from theoretical results in [9] and [10], we approximate the Whittle log-likelihood with a mixture of normal distributions, with frequency-dependent means and logistic weights. The implied prior model for $\log (f(\omega))$ enables a wide range of shapes for smooth spectral densities. The mixture representation of the log-spectral density is advantageous in that it facilitates extensions to hierarchical modeling in the multiple time series setting. The model described here is a starting point for such generalizations.

The outline of the paper is as follows. In Section 2, we describe the mixture modeling approach for spectral density estimation. In Section 3, we present results from synthetic and real datasets, and Section 4 provides concluding remarks.

\section{Mixture model for the spectral density}

[9] show that an exponential response distribution, involving a non-linear regression function, can be approximated by a mixture of exponential distributions with means that depend linearly (on the log scale) on the covariates and with covariate dependent mixture weights. Moreover, [10] presents approximation properties of finite mixtures of normal regressions as flexible models for conditional densities. The work in [10] focuses on the joint distribution of the response and covariates, showing that, under certain conditions, the joint distribution can be approximated in KullbachLeibler divergence by different specifications of finite mixtures of normals in which means, variances, and weights can depend on the covariates. Here, we consider fixed covariates, specifically the Fourier frequencies.

We thus propose to approximate the Whittle log-likelihood with a mixture of normal distributions, with frequency-dependent means and weights. We assume that $\log (f(\omega))$ 
and its first and second derivatives are continuous and bounded. In practice, this requirement translates into a smoothness assumption on the log-spectral density.

At the Fourier frequencies, for $j=1, . ., N$, we have $\mathrm{E}\left[\log \left(I_{n}\left(\omega_{j}\right)\right)\right]=\log \left(f\left(\omega_{j}\right)\right)-$ $\gamma$, where $\gamma$ is the Euler-Mascheroni constant. Let $y_{j}=\log \left(I_{n}\left(\omega_{j}\right)\right)+\gamma$. Under the Whittle likelihood approximation - that is, the exponential distribution for the $I_{n}\left(\omega_{j}\right)$ with mean $f(\omega)$ - the $y_{j}$ are independent with the following distribution:

$$
f_{Y}(y)=\exp \{y-\gamma-\log (f(\omega))-\exp (y-\gamma-\log (f(\omega)))\}, \quad y \in \mathbb{R} .
$$

Notice that the distribution in 11 is in the exponential family, and $-y_{j}$ are Gumbel distributed with scale parameter 1 and location parameter defined additively through $\log (f(\omega))$ and $\gamma$, such that the mean is $-\log (f(\omega))$. Although the Whittle approximate likelihood for either the $I_{n}\left(\omega_{j}\right)$ or the $y_{j}$ is based on standard distributions, the spectral density enters the likelihood in a non-standard fashion through the mean parameter. Our proposal is to apply a further approximation replacing the distribution in 11 with a structured mixture of normal distributions, which induces a flexible model for the $\log$-spectral density. The normal mixture approximation is motivated by the results in [10]. Alternatively, following [9], the model can be built from a mixture of exponential or Gumbel distributions on the original or log scale, respectively. Since convergence results can be obtained under all three mixture model formulations, we favor the normal mixture model as it results in a significantly less complex posterior simulation method.

We thus approximate the distribution of $y_{j}$ with a mixture of normal distributions, with means that depend linearly on $\omega_{j}$ and frequency-dependent logistic weights:

$$
y_{j} \mid \Theta \stackrel{i n d}{\sim} \sum_{k=1}^{K} g_{k}\left(\omega_{j} ; \lambda, \zeta, \phi\right) \mathrm{N}\left(y_{j} \mid \alpha_{k}+\beta_{k} \omega_{j}, \sigma^{2}\right), \quad j=1, \ldots, N
$$

where $g_{k}\left(\omega_{j} ; \lambda, \zeta, \phi\right)=\exp \left\{\left(\zeta_{k}+\phi_{k} \omega_{j}\right) / \lambda\right\} / \sum_{i=1}^{K} \exp \left\{\left(\zeta_{i}+\phi_{i} \omega_{j}\right) / \lambda\right\}$. Here, $\Theta$ collects all model parameters: the logistic weights parameters, $\zeta=\left\{\zeta_{k}: k=1, \ldots, K\right\}$ and $\phi=\left\{\phi_{k}: k=1, \ldots, K\right\}$, the intercept and slope parameters for the means of the normal mixture components, $\alpha=\left\{\alpha_{k}: k=1, \ldots, K\right\}$ and $\beta=\left\{\beta_{k}: k=1, \ldots, K\right\}$, as well as parameters $\lambda$ and $\sigma^{2}$. The logistic weights partition the support with soft boundaries. The parameter $\lambda$ controls the smoothness of the transition between the subsets 
of $(0, \pi)$ induced by the logistic weights. The larger the value of $\lambda$, the smoother is the corresponding estimate of the log-spectral density.

Under the approximation in (2), the model for the log-spectral density is given by

$$
\log (f(\omega))=\sum_{k=1}^{K} g_{k}(\omega ; \lambda, \zeta, \phi)\left(\alpha_{k}+\beta_{k} \omega\right) .
$$

Hence, the induced prior model for the log-spectral density admits a representation as a mixture of linear functions with component specific intercept and slope parameters, and with frequency-dependent weights that allow for local adjustment and thus flexible spectral density shapes, including non-linear log-spectral density estimates.

In addition to the appealing interpretation of the implied spectral density model, further theoretical justification for the approximation in (2) can be provided by means of results in the $L_{p}$ norm for the spectral density. The following result (the proof of which is given in Appendix A) establishes that the distance in the $L_{p}$ norm (denoted by $\|\cdot\|_{p}$ ) between the target log-spectral density, $h$, and the mixture model $h_{K}(\omega)=$ $\sum_{k=1}^{K} g_{k}(\omega ; \lambda, \zeta, \phi)\left(\alpha_{k}+\beta_{k} \omega\right)$ is bounded by a constant which is inversely proportional to the square of the number of mixture components $K$.

THEOREM. Let $h \in W_{2, K_{0}}^{\infty}$, that is, the Sobolev space of continuous functions bounded by $K_{0}$, with the first two derivatives continuous and bounded by $K_{0}$. Then, $\inf _{h_{K}}\left\|h_{K}-h\right\|_{p} \leq \pi^{2} K_{0} /\left(2 K^{2}\right)$, for any $p \geq 1$.

Therefore, the $L_{p}$ distance between the true log-spectral density and the proposed mixture is bounded by a constant proportional to $K_{0} / K^{2}$, where $K_{0}$ is related to the smoothness of the true spectral density. Hence, the distance decreases quadratically with the number of components. If we have prior knowledge on the smoothness of the log-spectral density, we can use it to fix $K$. For practical purposes, confirmed from empirical investigation with several data sets including the ones of Section 3, we have observed that in general a relatively small number of mixture components suffices to capture different spectral density shapes, with inference results being robust to the choice of $K$.

To complete the full Bayesian model, we assume prior independence among and between the parameters of each mixture component. Specifically, we use a normal prior distribution with mean $\mu_{\alpha}$ and variance $\sigma_{\alpha}^{2}$ for the $\alpha_{k}$, and a normal prior with mean $\mu_{\beta}$ 
and variance $\sigma_{\beta}^{2}$ for the $\beta_{k}$. For the common variance parameter, $\sigma^{2}$, we use an inversegamma prior, and for the smoothness parameter, $\lambda$, a gamma prior. We place standard normal priors on the $\zeta_{k}$ and $\phi_{k}$. This choice is motivated by the fact that the variances of $\zeta_{k}$ and $\phi_{k}$ and $\lambda$ cannot be estimated simultaneously. The prior of $\lambda$ expresses our belief on the degree of smoothness of the spectral density. As demonstrated with the data examples of Section 3, the smoothness parameter can be learned from the data. The prior on the intercept parameters $\alpha_{k}$ summarizes information about the spectral density value near $\omega=0$. Moreover, the prior on the slope parameters $\beta_{k}$ can be used to express beliefs about the shape of the spectral density. For instance, for multimodal spectral densities, we expect some $\beta_{k}$ to be positive and some negative, whereas for unimodal spectral densities, we expect all $\beta_{k}$ to have the same sign.

The model in (2) can be expanded in hierarchical form by introducing configuration variables $\left(r_{1}, \ldots, r_{N}\right)$, where each $r_{j}, j=1, \ldots, N$, has a discrete distribution with values in $\{1, \ldots, K\}$ :

$$
\begin{aligned}
& y_{j} \mid r_{j}, \alpha, \beta, \sigma^{2} \stackrel{i n d}{\sim} \mathrm{N}\left(y_{j} \mid \alpha_{r_{j}}+\beta_{r_{j}} \omega_{j}, \sigma^{2}\right), \quad j=1, \ldots, N \\
& r_{j} \mid \zeta, \phi, \lambda \stackrel{i n d}{\sim} \sum_{k=1}^{K} g_{k}\left(\omega_{j} ; \lambda, \zeta, \phi\right) \delta_{k}\left(r_{j}\right), \quad j=1, \ldots, N
\end{aligned}
$$

where $\delta_{k}(\cdot)$ denotes a point mass at $k$. We develop a Markov chain Monte Carlo (MCMC) posterior simulation algorithm, which is based almost exclusively on Gibbs sampling steps. Details can be found in Appendix B.

Note that the prior variance of the spectral density increases with $\omega$. To minimize this effect, when fitting the model we normalize the support $(0, \pi)$ of the spectral density to the unit interval; the results can be reported on the original scale through straightforward transformation.

In conclusion of the methodological development, we reiterate the two key advantages of the modeling approach. First, casting the spectral density estimation problem in a mixture modeling framework allows for relatively straightforward implementation of a Gibbs sampler for posterior inference. Moreover, the proposed model naturally leads to hierarchical extensions to efficiently consider multiple time series and borrow strength across them. 

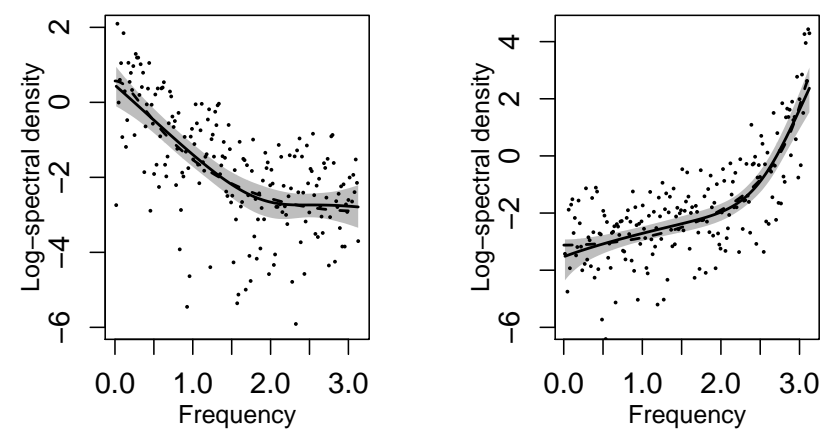

Figure 1: Synthetic data. Posterior mean (solid dark line), 95\% credible interval (shaded area), true log-spectral density (dashed line), and log-periodogram (dots) for data simulated from autoregressive processes of order one, with parameters 0.7 (left) and -0.9 (right).

\section{Numerical illustrations}

\subsection{Synthetic datasets}

We evaluate the performance of our method through four synthetic data examples. In all cases, for the $\alpha_{k}$ and $\beta_{k}$, we used zero-mean normal priors with variances $\sigma_{\alpha}^{2}=$ $\sigma_{\beta}^{2}=100$. An inverse-gamma prior with shape parameter 3 and mean 3 was placed on $\sigma^{2}$. For $\lambda$, we used a gamma prior with shape parameter 2 and mean 0.2 , which places almost all its mass in $(0,1)$. The number of mixture components was fixed at $K=10$; results were essentially the same with larger $K$.

Focusing first on monotonic spectral densities, we simulated data from two autoregressive (AR) processes of order one. Specifically, we consider $x_{1, t}=0.7 x_{1, t-1}+\epsilon_{1, t}$, with $\epsilon_{1, t} \sim \mathrm{N}(0,1)$ and $x_{2, t}=-0.9 x_{2, t-1}+\epsilon_{2, t}$, with $\epsilon_{2, t} \sim \mathrm{N}(0,1)$, and simulated 400 observations from each of these processes. The estimated log-spectral densities and the corresponding 95\% posterior intervals are reported in Figure 1 together with the true log-spectral densities and the periodogram observations. Our model successfully captures the monotonic trends and shapes of the underlying spectral densities. The posterior density for $\lambda$ for each case is plotted in Figure 3 

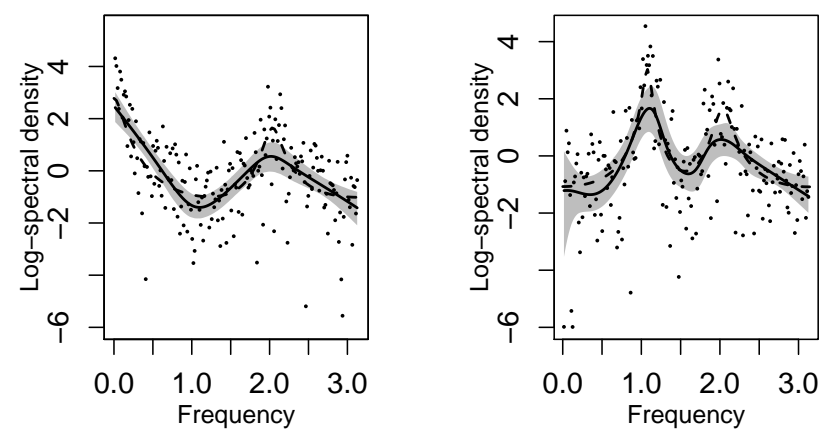

Figure 2: Synthetic data. Posterior mean (solid dark line), 95\% credible interval (shaded area), true log-spectral density (dashed line), and log-periodogram (dots) for the sum of autoregressive processes and white noise.

To evaluate model performance for more complex spectral densities, we simulated data from the sum of two AR processes and a white noise term. Specifically, let $x_{3, t}=$ $0.9 x_{3, t-1}+\epsilon_{3, t}$, with $\epsilon_{3, t} \sim \mathrm{N}(0,1), x_{4, t}=0.9 x_{4, t-1}-0.9 x_{4, t-2}+\epsilon_{4, t}$, with $\epsilon_{4, t} \sim$ $\mathrm{N}(0,1)$, and $x_{5, t}=-0.8 x_{5, t-1}-0.8 x_{5, t-2}+\epsilon_{5, t}$, with $\epsilon_{5, t} \sim \mathrm{N}(0,1)$. We construct $z_{1, t}=x_{3, t}+x_{5, t}+\nu_{1, t}$, where $\nu_{1, t} \sim \mathrm{N}(0,1)$, and $z_{2, t}=x_{4, t}+x_{5, t}+\nu_{2, t}$, where $\nu_{2, t} \sim \mathrm{N}(0,1)$. We simulated 400 observations from each of these two processes. The spectral density of $z_{1, t}$ is decreasing for low frequencies and has a peak around $\omega=2$ (Figure 2. left panel); the spectral density of $z_{2, t}$ is bimodal, with two peaks around $\omega=1.1$ and $\omega=2$ (Figure 2, right panel). The estimated log-spectral densities for the two processes along with $95 \%$ posterior intervals are reported in Figure 2 Our model successfully identifies the peaks of the true spectral densities and generally captures well their shape, especially considering that this is a harder problem than estimating the spectra of purely AR processes, as the AR structure here is latent rather than directly observed. The corresponding posterior densities for $\lambda$ are plotted in Figure 3 In both cases, the posterior distribution of $\lambda$ is supported by smaller values than for the AR processes of order one, in agreement with the fact that the spectral densities of $x_{1, t}$ and 

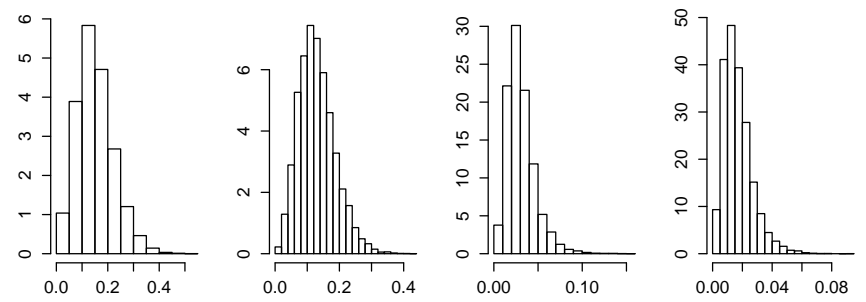

Figure 3: Synthetic data. Posterior density for $\lambda$ for data simulated from (from the left) autoregressive processes of order one, with parameter 0.7 and -0.9 , and for the sum of autoregressive processes and white noise.

$x_{2, t}$ are smoother than those of $z_{1, t}$ and $z_{2, t}$.

\subsection{Electroencephalogram data}

We consider four times series that correspond to portions of electroencephalograms taken from a larger dataset. The original time series were recorded at 19 locations over the scalp of a patient who received electroconvulsive therapy. Further details and data analysis can be found in [11]. The time series were recorded in four left channels, two of which are in the frontal region of the scalp (F7 and F3), one is in the temporal region (T5), and one is in the parietal region (P3). For each time series, we have 299 observations, obtained by subsampling the electroencephalogram signal every sixth observation from a mid-seizure section. The original sampling rate was $256 \mathrm{~Hz}$. The

priors were the same with Section 3.1, except for the prior for $\lambda$, that here is given by a gamma distribution with shape parameter 3 and mean 0.1 , which places almost all its mass in $(0,0.5)$. We choose this prior because we expect at least one pronounced peak in the spectral density, reflecting brain activity in at least one frequency band, and we thus want to avoid oversmoothing. The number of components is fixed to $K=10$; also in this case, the results were robust with respect to the number of components.

Figure 4 shows the posterior mean estimates and $95 \%$ posterior credible intervals for the spectral densities together with the logged spectral periodogram. All the channels show a peak around $3.4 \mathrm{~Hz}$. which is slightly shifted to the left in T5 and P3. These results are consistent with previous analyses which indicate that the observed quasi-periodicity is dominated by activity in the delta frequency range, that is, in the 
F7

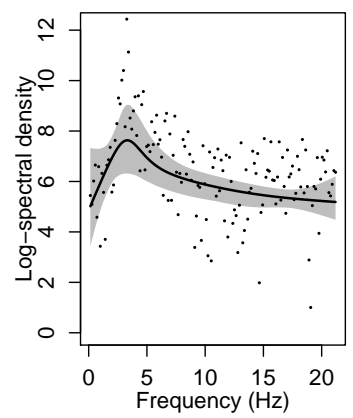

T5

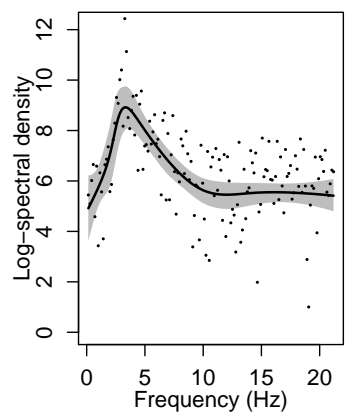

F3

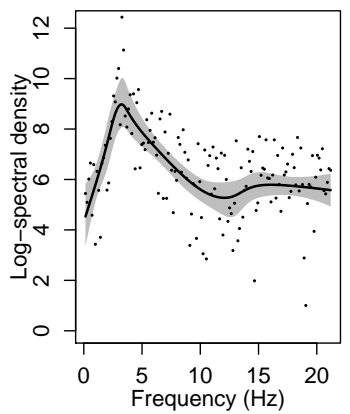

P3

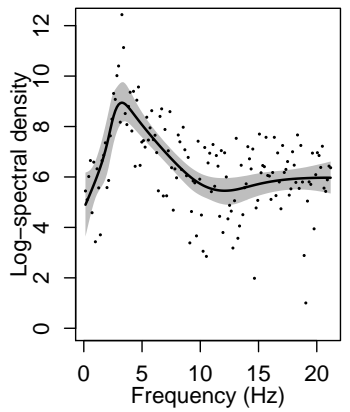

Figure 4: Posterior mean (solid dark line), 95\% credible interval (shaded area), and logperiodogram (dots) for the electroencephalogram datasets recorded in four channels.

range from 1 to $5 \mathrm{~Hz}$. We also note that although there are important similarities across the spectral densities, each density has its own features with channels F3, T5 and P3 sharing more similarities and F7 being different from the rest.

\section{Discussion}

We have presented a Bayesian approach to spectral density estimation that builds from an approximation to the Whittle log-likelihood through a mixture of normal distributions with linear means and frequency-dependent logistic weights. We have used simulated data examples to demonstrate the capacity of the model to uncover both monotonic and multimodal shapes for the underlying spectral density, with a fixed 
number of mixture components $K$. The model can be generalized with random $K$, albeit at the expense of more computationally challenging posterior simulation.

For certain types of non-Gaussian time series, it has been shown in [12] that the Whittle likelihood does not provide a satisfactory approximation for small sample sizes. An interesting direction for future study involves the applicability of the modeling approach for spectral densities of general non-Gaussian time series.

\section{Appendix A. Proof of the Theorem}

Let $\|f\|_{p}=\left(\int_{0}^{\pi}|f(\omega)|^{p} \mathrm{~d} P(\omega)\right)^{\frac{1}{p}}$ denote the $L_{p}$ norm, where $P$ is a distribution on $\Omega=[0, \pi]$ absolutely continuous with respect to Lebesgue measure. Moreover, denote by $\chi_{B}$ the indicator function for $B \subseteq \Omega$, and define the partition $\left\{Q_{1}^{K}, \ldots, Q_{K}^{K}\right\}$ of $\Omega$, where $Q_{k}^{K}=[(k-1) \pi / K, k \pi / K)$, for $k=1, \ldots, K-1$, and $Q_{K}^{K}=[(K-1) \pi / K, \pi]$. The following lemma from [9] is used to prove the main result.

Lemma For logistic weights, $g_{k}(\omega ; \lambda, \zeta, \phi), k=1, \ldots, K$, as defined in (3), we have that, for all $K$ and for each $\epsilon>0$, there exist $\zeta^{(\epsilon)}, \phi^{(\epsilon)}, \lambda^{(\epsilon)}$ such that:

$\sup _{1 \leq k \leq K}\left\|g_{k}\left(\cdot ; \lambda^{(\epsilon)}, \zeta^{(\epsilon)}, \phi^{(\epsilon)}\right)-\chi_{Q_{k}^{K}}(\cdot)\right\|_{p}<\epsilon$.

Proof of Theorem. The proof is along the lines of the one in [13]. We start by proving that, for fixed $K$, any $h \in W_{2, K_{0}}^{\infty}$ can be approximated by a piecewise linear function on the partition $\left\{Q_{1}^{K}, \ldots, Q_{K}^{K}\right\}$, with the $L_{p}$ distance bounded by a constant that depends on $K$. For each interval $Q_{k}^{K}$, consider a point $\omega_{k}^{*} \in Q_{k}^{K}$ and the linear approximation based on the first-order Taylor series expansion: $\hat{h}_{k}(\omega)=\hat{\alpha}_{k}+\hat{\beta}_{k} \omega$, for $\omega \in Q_{k}^{K}$, where $\hat{\alpha}_{k}=h\left(\omega_{k}^{*}\right)-\omega_{k}^{*} h^{\prime}\left(\omega_{k}^{*}\right)$ and $\hat{\beta}_{k}=h^{\prime}\left(\omega_{k}^{*}\right)$; here, $h^{\prime}\left(\omega_{k}^{*}\right)$ denotes the first derivative of $h(\omega)$ evaluated at $\omega_{k}^{*}$, with similar notation used below for the second derivative. We have $\left\|\left\{\sum_{k=1}^{K} \chi_{Q_{k}^{K}} \hat{h}_{k}\right\}-h\right\|_{p}=\left\|\sum_{k=1}^{K} \chi_{Q_{k}^{K}}\left\{\hat{h}_{k}-h\right\}\right\|_{p} \leq$ $\sup _{1 \leq k \leq K}\left\|\hat{h}_{k}-h\right\|_{\infty}$, where $\|\cdot\|_{\infty}$ denotes the $L_{\infty}$ norm. Now, for each interval $Q_{k}^{K}$, we consider the second-order expansion of $h(\omega)$ around the same $\omega_{k}^{*} \in Q_{k}^{K}$. Note that the partition $\left\{Q_{1}^{K}, \ldots, Q_{K}^{K}\right\}$ satisfies the property that, for any $k$, and for any $\omega_{1}$ and $\omega_{2}$ in $Q_{k}^{K},\left|\omega_{1}-\omega_{2}\right| \leq \pi / K$. Using this property and the fact that the second derivative of $h$ is bounded by $K_{0}$, we obtain $\left|\hat{h}_{k}(\omega)-h(\omega)\right| \leq\left|0.5\left(\omega-\omega_{k}^{*}\right)^{2} h^{\prime \prime}\left(\omega_{k}^{*}\right)\right| \leq$ 
$\pi^{2} K_{0} /\left(2 K^{2}\right)$. Therefore, $\left\|\left\{\sum_{k=1}^{K} \chi_{Q_{k}^{K}} \hat{h}_{k}\right\}-h\right\|_{p} \leq \pi^{2} K_{0} /\left(2 K^{2}\right)$.

To simplify notation, let $g_{k} \equiv g_{k}(\cdot ; \lambda, \zeta, \phi)$. Using the triangular inequality, we can write $\left\|\left\{\sum_{k=1}^{K} g_{k} \hat{h}_{k}\right\}-h\right\|_{p} \leq\left\|\sum_{k=1}^{K}\left\{g_{k}-\chi_{Q_{k}^{K}}\right\} \hat{h}_{k}\right\|_{p}+\left\|\left\{\sum_{k=1}^{K} \chi_{Q_{k}^{K}} \hat{h}_{k}\right\}-h\right\|_{p}$. Based on the previous result, the second term is bounded by $\pi^{2} K_{0} /\left(2 K^{2}\right)$. For the first term, we have $\left\|\sum_{k=1}^{K}\left\{g_{k}-\chi_{Q_{k}^{K}}\right\} \hat{h}_{k}\right\|_{p} \leq \sum_{k=1}^{K}\left\|g_{k}-\chi_{Q_{k}^{K}}\right\|_{p}\left\|\hat{h}_{k}\right\|_{\infty}$. Using the lemma and the fact that $\left|\hat{h}_{k}(\omega)\right| \leq\left|h\left(\omega_{k}^{*}\right)\right|+\left|h^{\prime}\left(\omega_{k}^{*}\right)\left(\omega-\omega_{k}^{*}\right)\right| \leq K_{0}+\pi K_{0}$, we have that the first term is bounded by $K \epsilon(1+\pi) K_{0}$. Finally, $\left\|\left\{\sum_{k=1}^{K} g_{k} \hat{h}_{k}\right\}-h\right\|_{p} \leq$ $K \epsilon(1+\pi) K_{0}+\left\{\pi^{2} K_{0} /\left(2 K^{2}\right)\right\}$, and letting $\epsilon$ tend to zero, we obtain the result.

\section{Appendix B. Full conditional distributions for the Gibbs sampler algorithm}

We generate samples from the posterior distribution of the model in 4, using an MCMC algorithm with Gibbs sampling steps and one Metropolis step for $\lambda$. The MCMC algorithm comprises the following updates:

- For $j=1, \ldots, N$, sample $r_{j}$ from a discrete distribution on $\{1, \ldots, K\}$ with probabilities proportional to $g_{k}\left(\omega_{j} ; \lambda, \zeta, \phi\right) \mathrm{N}\left(y_{j} \mid \alpha_{k}+\beta_{k} \omega_{j}, \sigma^{2}\right)$, for $k=1, \ldots, K$.

- For $k=1, \ldots, K$, sample $\left(\alpha_{k}, \beta_{k}\right)^{\prime}$ from a bivariate normal distribution with covariance matrix $\Sigma^{*}=\left(\sigma^{-2} \sum_{\left\{j: r_{j}=k\right\}} z_{j} z_{j}^{\prime}+\Sigma_{0}^{-1}\right)^{-1}$ and mean $\mu^{*}=\Sigma^{*}\left(\Sigma_{0}^{-1} \mu_{0}+\right.$ $\left.\sum_{\left\{j: r_{j}=k\right\}} y_{j} z_{j}\right)$. Here, $z_{j}=\left(1, \omega_{j}\right)^{\prime}, \mu_{0}=\left(\mu_{\alpha}, \mu_{\beta}\right)^{\prime}$ is the prior mean, and $\Sigma_{0}$ the diagonal prior covariance matrix with diagonal elements $\sigma_{\alpha}^{2}$ and $\sigma_{\beta}^{2}$.

- Sample $\sigma^{2}$ from an inverse-gamma with parameters $n_{0}+N / 2$ and $d_{0}+\left\{\sum_{j=1}^{N}\left(y_{j}-\right.\right.$ $\left.\left.\left(\alpha_{r_{j}}+\beta_{r_{j}} \omega_{j}\right)\right)^{2}\right\} / 2$, where $n_{0}$ and $d_{0}$ are the parameters of the inverse-gamma prior.

- In order to sample $\left(\zeta_{k}, \phi_{k}\right)^{\prime}$, for $k=1, \ldots, K$, we use a data augmentation technique. For each $k$, we introduce $N$ Pólya-Gamma random variables, $q_{j}, j=$ $1, \ldots, N$, as defined in [14]. For $k=1, \ldots, K$, let $\psi_{k j}=\left(\zeta_{k}+\phi_{k} \omega_{j}\right) / \lambda$ and $c_{k j}=$ $\log \left(\sum_{\{i \neq k\}} \exp \left\{\left(\zeta_{i}+\phi_{i} \omega_{j}\right) / \lambda\right\}\right)$. Moreover, let $n_{k}$ be the number of $r_{j}$ such that $r_{j}=k$. Then, the $q_{j}$ are drawn from a Pólya-Gamma distribution with parameters 1 and $\psi_{k j}-c_{k j}$. And, given the $q_{j}$, we sample $\left(\zeta_{k}, \phi_{k}\right)^{\prime}$ from a bivariate normal with covariance matrix $\Sigma_{k}^{*}=\left(\mathbb{I}+\sum_{j=1}^{N} \lambda^{-2} z_{j} z_{j}^{\prime} q_{j}\right)^{-1}$, where $\mathbb{I}$ is the identity matrix, and mean $\mu_{k}^{*}=\Sigma_{k}^{*}\left(\sum_{\left\{j: r_{j}=k\right\}} \lambda^{-1} z_{j} c_{k j} q_{j}+\sum_{\left\{j: r_{j}=k\right\}} 0.5 \lambda^{-1} z_{j}-\sum_{\left\{j: r_{j} \neq k\right\}} 0.5 \lambda^{-1} z_{j}\right)$.

- Update $\lambda$ with a Gaussian random-walk Metropolis step on $\log (\lambda)$. 


\section{Acknowledgement}

This research was supported in part by the National Science Foundation under award DMS 1407838. The authors wish to thank two reviewers for useful comments.

\section{References}

[1] E. Parzen, On estimation of a probability density function and mode, The Annals of Mathematical Statistics 33 (3) (1962) 1065-1076.

[2] P. Whittle, Curve and periodogram smoothing, Journal of the Royal Statistical Society, Series B 19 (1957) 38-63.

[3] G. Wahba, Automatic smoothing of the log periodogram, Journal of the American Statistical Association 75 (369) (1980) 122-132.

[4] Y. Pawitan, F. O'Sullivan, Nonparametric spectral density estimation using penalized Whittle likelihood, Journal of the American Statistical Association 89 (426) (1994) 600-610.

[5] C. K. Carter, R. Kohn, Semiparametric Bayesian inference for time series with mixed spectra, Journal of the Royal Statistical Society, Series B 59 (1) (1997) $255-268$.

[6] O. Rosen, D. Stoffer, Automatic estimation of multivariate spectra via smoothing splines, Biometrika 94 (2007) 335-345.

[7] N. Choudhuri, S. Ghosal, A. Roy, Bayesian estimation of the spectral density of a time series, Journal of the American Statistical Association 99 (2004) 1050-1059.

[8] M. Pensky, B. Vidakovic, D. DeCanditiis, Bayesian decision theoretic scaleadaptive estimation of a log-spectral density, Statistica Sinica 17 (2007) 635-666.

[9] W. Jiang, M. A. Tanner, Hierarchical mixtures-of-experts for exponential family regression models: Approximation and maximum likelihood estimation, The Annals of Statistics 27 (1999) 987-1011. 
[10] A. Norets, Approximation of conditional densities by smooth mixtures of regressions, The Annals of Statistics 38 (2010) 1733-1766.

[11] A. Krystal, R. Prado, M. West, New methods of time series analysis of nonstationary EEG data: eigenstructure decompositions of time-varying autoregressions, Clinical Neurophysiology 110 (1999) 2197-2206.

[12] A. Contreras-Cristan, E. Gutierrez-Pena, S. G. Walker, A note on whittle's likelihood, Communications in Statistics - Simulation and Computation 35 (4) (2006) $857-875$.

[13] W. Jiang, M. A. Tanner, On the approximation rate of hierarchical mixtures-ofexperts for generalized linear models, Neural computation 11 (5) (1999) 11831198.

[14] N. G. Polson, J. G. Scott, J. Windle, Bayesian inference for logistic models using Pólya-Gamma latent variables, Journal of the American Statistical Association 108 (504) (2013) 1339-1349. 